\title{
Mechanism involved in the modulation of photoreceptor-specific cyclic nucleotide- gated channel by the tyrosine kinase adapter protein Grb14
}

\author{
Vivek K. Gupta ${ }^{1,4}$, Ammaji Rajala ${ }^{1,4}$, Karla K. Rodgers ${ }^{3}$, Raju V. S. Rajala ${ }^{1,2,4} \bowtie$ \\ 1 Department of Ophthalmology, University of Oklahoma Health Sciences Center, Oklahoma City, OK 73104, USA \\ 2 Department of Cell Biology, University of Oklahoma Health Sciences Center, Oklahoma City, OK 73104, USA \\ 3 Department of Biochemistry and Molecular Biology, University of Oklahoma Health Sciences Center, Oklahoma City, \\ OK 73104, USA \\ 4 Dean A. McGee Eye Institute, University of Oklahoma Health Sciences Center, Oklahoma City, OK 73104, USA \\ $\triangle$ Correspondence: raju-rajala@ouhsc.edu
}

Received September 20, 2011 Accepted October 16, 2011

\section{ABSTRACT}

We recently found that growth factor receptor-bound (Grb) protein 14 is a novel physiological modulator of photoreceptor specific cyclic nucleotide-gated channel alpha subunit (CNGA1). Grb14 promotes the CNG channel closure through its Ras-associating (RA) domain. In the current study we show that this RA domain-mediated inhibition of rod CNG channel is electrostatic in nature. Grb14 competes with cGMP for the CNGA1 binding pocket and electrostatically interacts with $\mathrm{Arg}^{559}$ through a negatively charged $\beta$-turn at its RA domain. Moreover, the three Glu residues (180-182) in Grb14 are absolutely critical for electrostatic interaction with the cGMP binding pocket and resultant inhibition. Our study also demonstrates that substitution of Lys 140 for Ala or in combination with polyglutamte mutants of Grb14 results in a significantly reduced binding with CNGA1. These results suggest that in addition to Glu $^{180-182}$ and Lys ${ }^{140}$, other residues in Grb14 may be involved in the electrostatic interaction with CNGA1. The RA domain is highly conserved among the members of Grb7 family of proteins, which includes Grb7, Grb10 and Grb14. Further, only Grb14 is able to modulate the channel activity, but not Grb7 and Grb10. All together, it suggests the existence of a divergence in RA domains among the members of the Grb7 family.
(Grb14), Ras-associating domain, cyclic nucleotide gated channel, rod outer segments, tyrosine kinase signaling

\section{INTRODUCTION}

Growth factor receptor-bound protein 14 (Grb14) is a member of an emerging family of noncatalytic adapter proteins, which also includes Grb7 and Grb10 (Daly, 1998; Holt and Siddle, 2005). It is a multidomain protein that possesses several intracellular signaling modules including a Ras-associating domain (RA), a Pleckstrin homology $(\mathrm{PH})$ domain, a BPS (Between $\mathrm{PH}$ and $\mathrm{SH} 2$ ) and a C-terminal $\mathrm{SH} 2$ domain, as well as a conserved $\mathrm{N}$-terminal proline rich motif (Daly et al., 1996). We discovered that Grb14, an adapter molecule involved in tyrosine kinase signaling, undergoes lightdependent translocation within rod photoreceptor cells (Rajala et al., 2009). Grb14 is localized predominantly to the inner segment, nuclear layer and synapse in dark-adapted rods, whereas in the light-adapted rods Grb14 is distributed throughout the entire cell, including the outer segment (Rajala et al., 2009). Very recently we have demonstrated that lightdependent translocated Grb14 binds to cyclic nucleotidegated channel alpha (CNGA1) subunit in outer segments through its RA domain (Gupta et al., 2010). We have further demonstrated that in the Grb14 ${ }^{-1-}$ mice, the channel remains opened in light, suggesting that Grb14 is a normal physiological modulator of CNG channel in vivo (Gupta et al., 2010); however, the molecular determinants of Grb14-mediated channel modulation are not known. CNG channels play 
important roles in visual and olfactory transduction (Yau and Baylor, 1989; Finn et al., 1996; Biel et al., 1999; Kaupp and Seifert, 2002; Yau and Hardie, 2009; Zhang et al., 2009). In the visual system, a cyclic guanosine monophosphate (cGMP)-gated channel is found in the outer membrane of retinal photoreceptor cells. The cGMP-sensitive cation channel is the sensor of the changes in cytoplasmic cGMP concentration brought about by light (Yau and Baylor, 1989). The dynamic interaction between Grb14 and CNGA1 promotes the channel to be in an off state, through a competitive binding to the cGMP binding site (Gupta et al., 2010). Grb14 interacts with CNGA1 in a competitive manner and increases the Michaelis-Menten constant $\left(K_{m}\right)$ of cGMP for the CNGA1. The kinetic studies unambiguously indicated that Grb14 binds to or interferes with the cGMP binding site of CNGA1 and that its inhibitory effect can be greatly alleviated by increasing the concentration of CGMP. Here we characterize in detail the molecular mechanism underlying the RAGrb14 interaction with the C-terminal region (CTR) of CNGA1. The RA-Grb14 and CTR-CNGA1 interaction is electrostatic in nature. The role of individual residues in this functional and physical interaction has been elucidated in the current study by a combination of molecular modeling, site-directed mutagenesis and secondary structural analysis. The role of relevant residues in influencing the activity of CNG channel as well as the theory underlying these binding interactions has been studied. The Grb14 belongs to the Grb7 family and is a rapidly emerging group of Src homology $(\mathrm{SH}) 2$ domaincontaining signaling proteins that currently contains three members, Grb7, 10 and 14. Even though the RA domain is highly conserved among the members of Grb7 family, only Grb14 is able to modulate the channel activity, but not Grb7 and Grb10. These studies suggest the existence of divergence in RA domains among the members of the Grb7 family.

\section{RESULTS}

\section{Spatially constrained electrostatic interactions with Grb14 result in modulation of the cGMP sensitivity of CNGA1}

Our earlier study suggests that Grb14 inhibits cGMP binding competitively and promotes the channel to be in a closed conformation, and that high concentrations of cGMP relieve the Grb14-mediated inhibition of the channel (Gupta et al., 2010). The cGMP binding pocket has been studied extensively (Kumar and Weber, 1992; Kaupp and Seifert, 2002). The cGMP binding domain of CNG channel was modeled using [PDB id. 1Q3E] as the template (Fig. 1B). The critical phosphate binding region between strands beta 6 and 7 was followed for energy minimization and any side-chain conflicts (Vriend, 1990; Morris et al., 1992; Guex and Peitsch, 1997). The RA domain of Grb14 was modeled using RA-Grb10 (Depetris et al., 2009) (3HKO) as the template and was investigated for any negatively charged topological surfaces. Topological analysis revealed that the RA domain carries a singular highly negatively charged poly-Glu sequence (180-182) placed on the $\beta$-turn, which is well exposed and surface accessible (Fig. 1A).

This sequence (E180-182Q) was the most promising candidate for analyses of potential interactions with $\mathrm{Arg}^{559}$ based on geometrical orientation and accessibility. Molecular modeling studies revealed that $\mathrm{Glu}^{180}$ is geometrically positioned perpendicular to $\mathrm{Glu}^{181}$ and can form a potential salt bridge formation with $\mathrm{Arg}^{559}$. The $\mathrm{Glu}^{180}$ side chain is directed inwardly to a chamber through which the cGMP is translocated. The Glu residues are located on a flexible and protruded $\beta$-turn, with their side chains extended perpendicular to each other, thus providing ample length to span the distance between the brim and the side chain of the $\mathrm{Arg}^{559}$ $(7 \AA)$ constituting the terminal end of the cavity. $\mathrm{Glu}^{181}$ may help position $\mathrm{Glu}^{180}$ in a proper perspective by interacting with another positively charged exposed neighbor Lys ${ }^{506}$ and thereby providing an additional anchorage (Fig. 1C and 1D). Lys ${ }^{506}$ was found to line the "cavity" proximal to $\mathrm{Arg}^{559}$ in CNGA1. Its orientation is such that it can undergo a favorable electrostatic interaction with $\mathrm{Glu}^{181}$. Lys ${ }^{506}$ can probably introduce a stabilizing interaction to the Grb14 and CNGA1. This orientation would lead to the prediction that Lys ${ }^{506}$ contributes a certain degree of strength and positioning to the anchorage of Glu residues by electrostatically interacting with $\mathrm{Glu}^{181}$. This interaction would mechanically obstruct the cGMP binding site of CNGA1 and thereby increase its $K_{\mathrm{m}}$ for CGMP.

The theoretical prediction was experimentally investigated by site-directed mutagenesis analysis. In accordance with the previous reports that $\mathrm{Arg}^{559}$ in CNGA1 is essential for cGMP sensitivity in the linear range and at physiological concentrations (Kumar and Weber, 1992; Liu et al., 1998), we found in the present study that substitution of $\mathrm{Arg}^{559}$ for GIn resulted in the loss of channel sensitivity towards CGMP and the sensitivity remains unchanged upon co-expression with wild type Grb14 (Fig. 2A). As predicted, the negatively charged polyglutamates (E180-182) in Grb14 are important for interaction with $\mathrm{Arg}^{559}$ in CNGA1 and substitution of Glu 180, 181 and 182 for Gln failed to modulate the channel activity (Fig. 2A). Similar results were observed when the mutant RA domain (E180-182Q) of Grb14 was co-expressed with wild type CNGA1 (Fig. 2A). To rule out the possibility that loss of Grb14-mediated suppression of CNGA1 function is due to overexpression of CNGA1 in the presence of mutant Grb14, transfected proteins were immunoblotted with Myc (for CNGA1, Grb14 and RA-Grb14) antibody. No difference in the expression of proteins was found (Fig. 2B), suggesting that channel modulation may be mediated through the molecular interaction between $\mathrm{Arg}^{559}$ in CNGA1 and polyglutamates in the RA domain of Grb14.

To establish whether the loss of mutant Grb14-mediated 

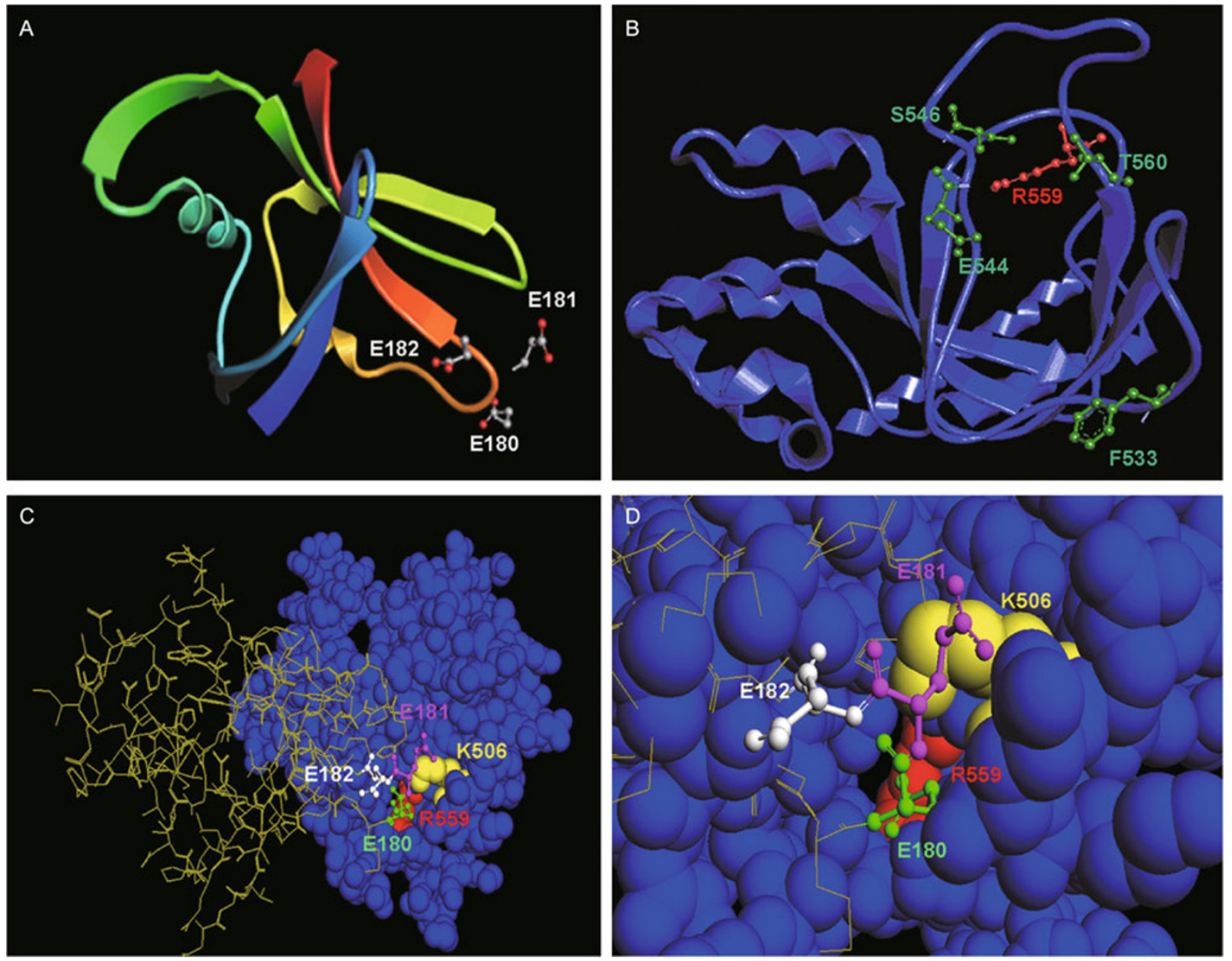

Figure 1. Structural basis for the Grb14-induced modulation of CNGA1 activity. Ribbon-shaped model of RA domain of Grb14 [UniProtKB Accession No. Q5ICW4] (106-192) constructed using the RA domain of Grb10 as the template (PDB ID: 3HK0). The Glu ${ }^{180-182}$ comprising a highly negatively charged protruded loop are highlighted as ball and stick representation (A). Ribbon model of CNGA1 (residues 400-600) (blue) based on the template [PDB ID. 1Q3E]. The $\operatorname{Arg}^{559}$ (red, ball and stick) located in a crevice, which can potentially interact with cyclic phosphate ring of cGMP, is highlighted and labeled. Ser ${ }^{546}, \mathrm{Glu}^{544}, \mathrm{Thr}^{560}$ and Phe ${ }^{533}$ residues comprising the binding pocket of CGMP are highlighted as ball and stick (green) and labeled (B). Complete view of the way RA domain of Grb14 (wireframe, light yellow) may interact with the cytoplasmic domain of CNGA1 (CPK, blue) (C). The negatively charged hair-pin loop of RA-Grb14 with projecting Glu side chains can interact with $\operatorname{Arg}^{559}$ (Red, labeled) placed in the cGMP binding pocket. The RA-Grb14 residues involved in this potential interaction are highlighted as ball and stick and labeled (Green: Glu ${ }^{180}$, Pink: Glu ${ }^{181}$ and White: $\mathrm{Glu}^{182}$ ) while the CNGA1 interacting residues are represented as space-filled structures (red: $\operatorname{Arg}^{559}$ and yellow: Lys ${ }^{506}$ ) and labeled. An enlarged view of the cGMP binding pocket providing the detailed geometry of interaction of Glu residues of Grb14 with Arg and Lys residues of CNGA1 (D). The color and representation scheme is the same as C.

inhibition of channel activity is due to the inability of the mutant Grb14 to interact with the channel or loss of interaction between $\mathrm{Arg}^{559}$ and $\mathrm{Glu}^{180-182}$, we immunoprecipitated the proteins with anti-CNGA1 antibody and examined for the association of wild type and mutant Grb14 (E180-182Q). The results indicated that irrespective of mutations, the CNGA1 is able to associate with full length wild type and mutant Grb14, as well as mutant RA-Grb14 (Fig. 2C). These experiments suggest that the molecular interaction between $\operatorname{Arg}^{559}$ in CNGA1 and polyglutamates (180-182) in the RA domain of Grb14 are responsible for channel modulation but are not critical for their binding. Since the RA-PH region of Grb14 constitutes a single structural unit (Gupta et al., 2010), the RA-PH wild type and mutant domains were investigated for their potential effects on CNGA1. Similar results were obtained as with RA domain alone in terms of both activity modulation (Fig. 3A) and physical binding (Fig. 3B and 3C). As observed previously, both the wild type and the mutant domains (E180-182Q) interacted and co-immunoprecipitated along with CNGA1 indicating that the interaction is not dictated by these three Glu residues and that there are other regions in the domain which significantly contribute 


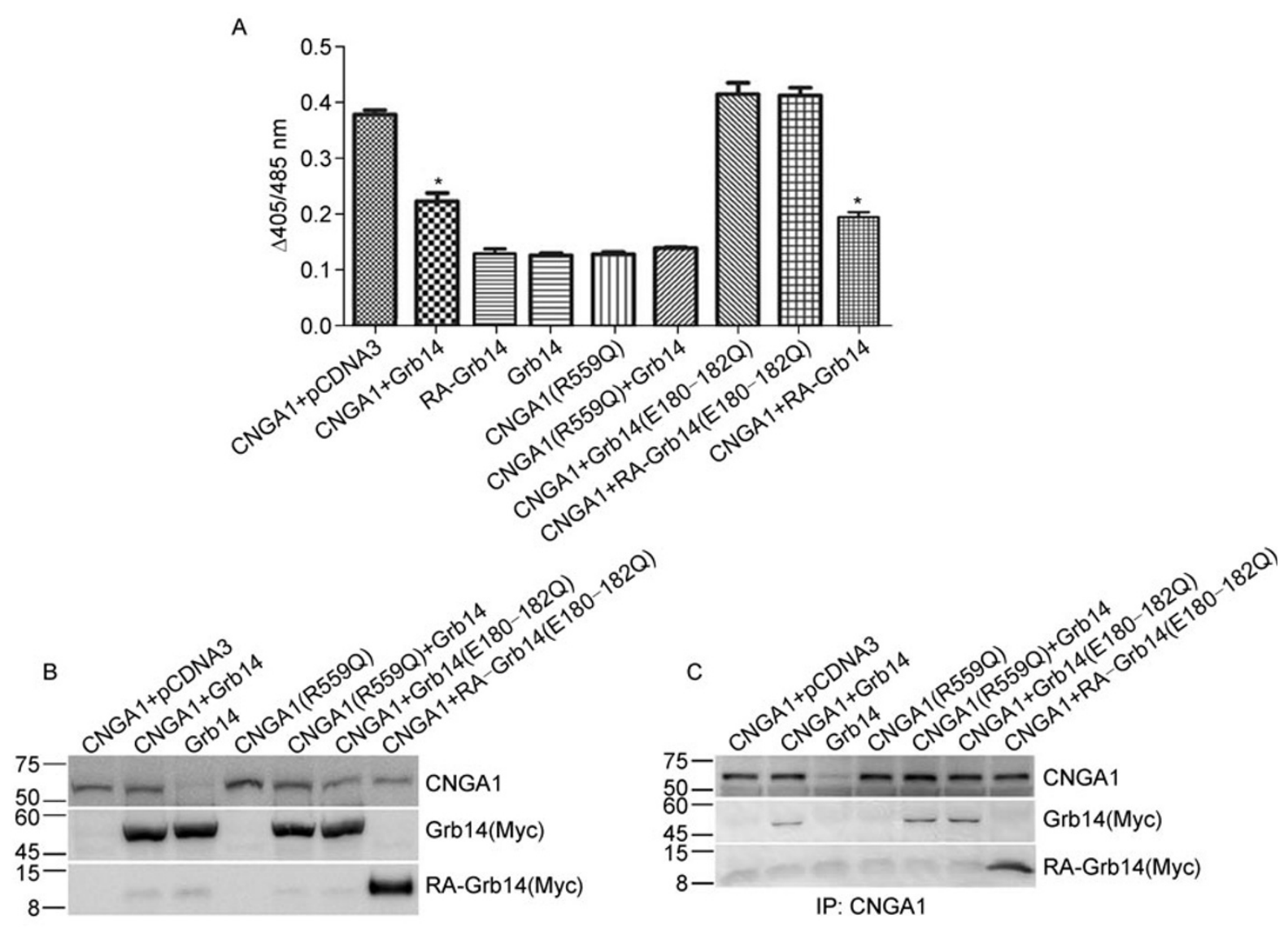

Figure 2. Polyglutamate sequence on the RA region of Grb14 is essential for modulation of CNGA1 activity but not for physical binding. Wild type or mutant CNGA1 (R559Q) was co-expressed with wild type or mutant (E180-182Q) myc-tagged Grb14 or myc-tagged RA domain of either wild type or mutant Grb14 (E180-182Q) in HEK293T cells and examined for their effects on Ca ${ }^{2+}$ influx in response to cGMP (A). The data are shown as mean $\pm \mathrm{SD}, n=3$ independent transfections. $* p<0.001$ (CNGA1 vs CNGA1 plus Grb14 or RA-Grb14). Protein expression in cellular lysates was determined by immunoblotting with Myc antibody for CNGA1, Grb14 and RA-Grb14 (B) or immunoprecipitated with anti-CNGA1 antibody and immunoblotted with Myc antibody and reprobed with anti-CNGA1 antibody (C).

towards this physical interaction. These studies suggest that channel modulation may be mediated through the molecular interaction between $\mathrm{Arg}^{559}$ in CNGA1 and polyglutamates in the RA domain of Grb14.

\section{Analysis of polyglutamate (E180-182) region for its role in binding to CNGA1 and its modulation}

To investigate whether the polyglutamate (E180-182) sequence in RA domain is the minimum stretch present at the $\beta$-turn that plays a role in modulation of CNG channel, or if a much smaller negative region might be sufficient, sitedirected mutagenesis was carried out with different combinations of these Glu residues. The co-expression of these constructs along with the CNGA1 channel illustrates that (E180-182) polyglutamate sequence is the minimum sequence that should be present in the right context of the
RA domain in order to possess the CNG channel modulatory properties (Fig. 4A). The expression profile of the proteins reveals that the lack of modulation was not due to the lack of either expression (Fig. 4B) or interaction of these mutant RA domains with CNGA1 (Fig. $2 \mathrm{C}$ and $3 \mathrm{C}$ ). We were unable to stably express E181Q mutant. Its role was instead investigated by expressing a double mutant of E180,181Q. These experiments suggest that RA domain is necessary and sufficient to modulate the channel activity, further confirming our earlier studies (Gupta et al., 2010).

\section{Glu residues in RA domain are essential for Grb14-mediated modulation of native rod CNG channel}

The effect of Grb14 on the cGMP-gated native channel activity was investigated using a fluorometric calcium ion influx assay (Gupta et al., 2010). The calcium sensitive dye, 


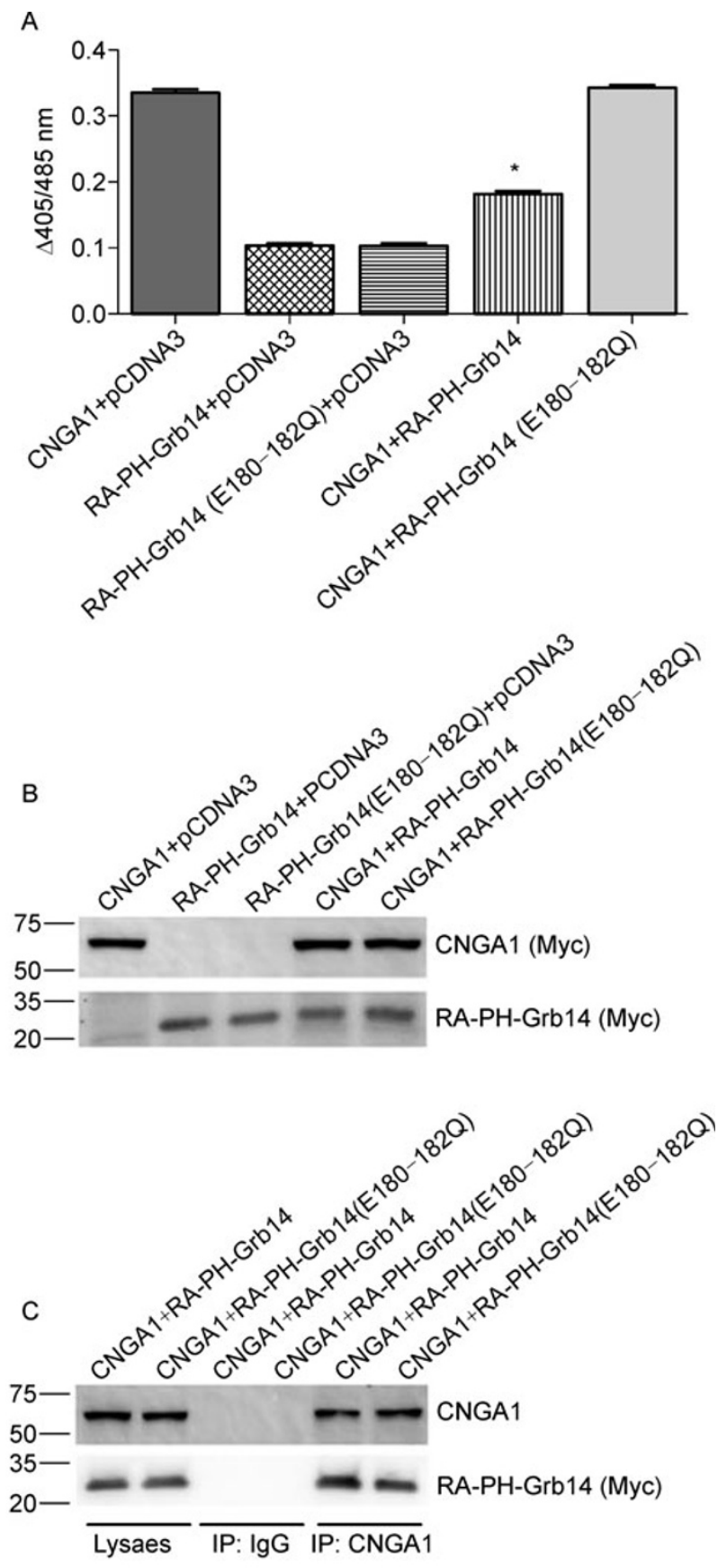

Figure 3. Polyglutamate sequence on the structurally distinct RA-PH domain of Grb14 is essential for modulation of CNGA1 activity but not for physical binding. RA-PH-Grb14 wild type or mutant was co-expressed with myc CNGA1 in HEK293T cells and examined for their effects on $\mathrm{Ca}^{2+}$ influx in response to cGMP (A). Control experiments include the expression of CNGA1 with pCDNA3 or the expression of wild type or mutant RA-PH-Grb14 alone. The data are shown as mean $\pm \mathrm{SD}, n=3$ independent transfections. $* p<0.001$ (CNGA1 vs CNGA1 plus RA-PH-Grb14). Protein expression in cellular lysates was determined by immunoblotting with Myc antibody for CNGA1 and RA-PH-Grb14 (B) or immunoprecipitated with anti-CNGA1 antibody and immunoblotted with Myc antibody and reprobed with anti-CNGA1 antibody (C).

Fluo-3, was trapped inside the washed rod outer segment (ROS) membrane vesicles and the rate of cGMP-mediated $\mathrm{Ca}^{2+}$ influx was monitored either in the presence of exogenously added recombinant GST-RA-Grb14 or GST alone (control) (Fig. 5B). The washed bovine ROS vesicles were made as described previously (Hsu and Molday, 1993). 

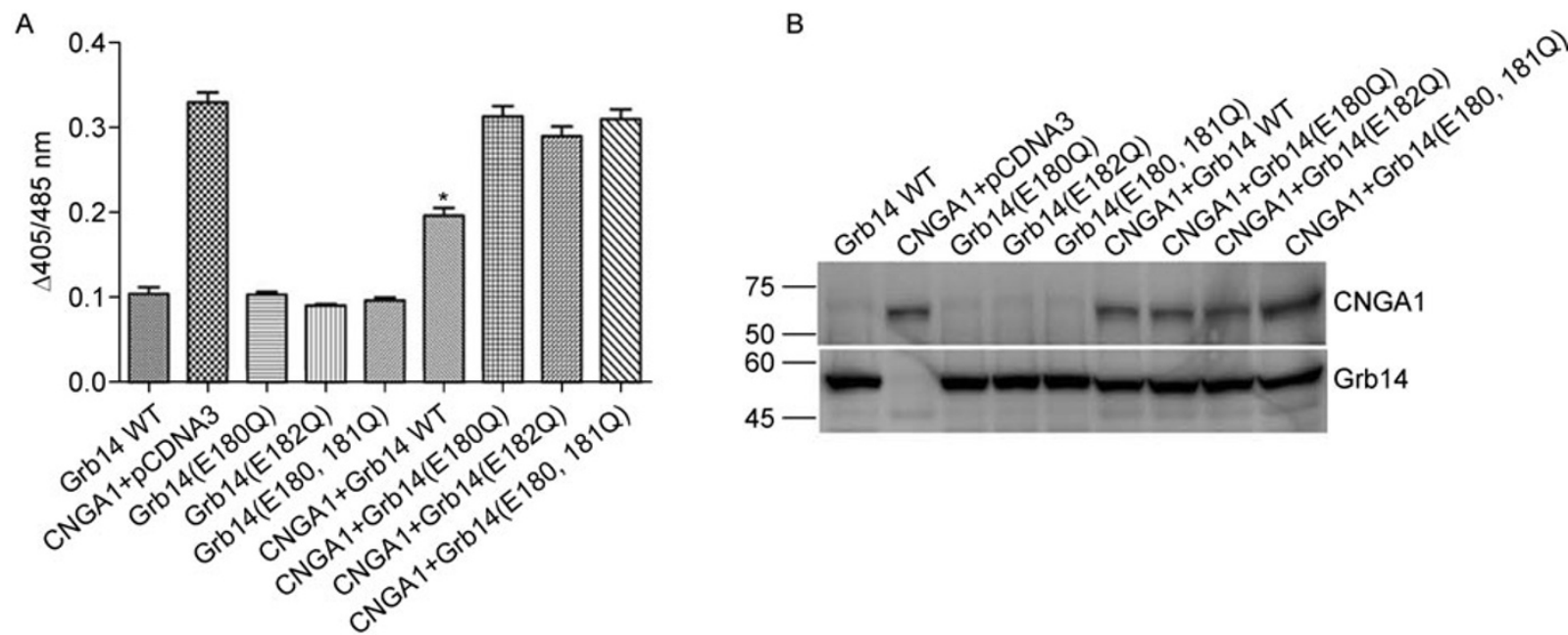

Figure 4. The three consecutive Glu residues in (Glu)3 sequence are necessary for the modulation of CNGA1 activity. Individual and double mutants of Grb14 as indicated were co-expressed in HEK293T cells and examined for their effects on Ca ${ }^{2+}$ influx in response to cGMP (A). The data are shown as mean $\pm \mathrm{SD}, n=3$ independent transfections. * $p<0.001$ (CNGA1 vs CNGA1 plus Grb14). Protein expression in cellular lysates was determined by immunoblotting with Myc antibody for CNGA1 and Grb14 (B).
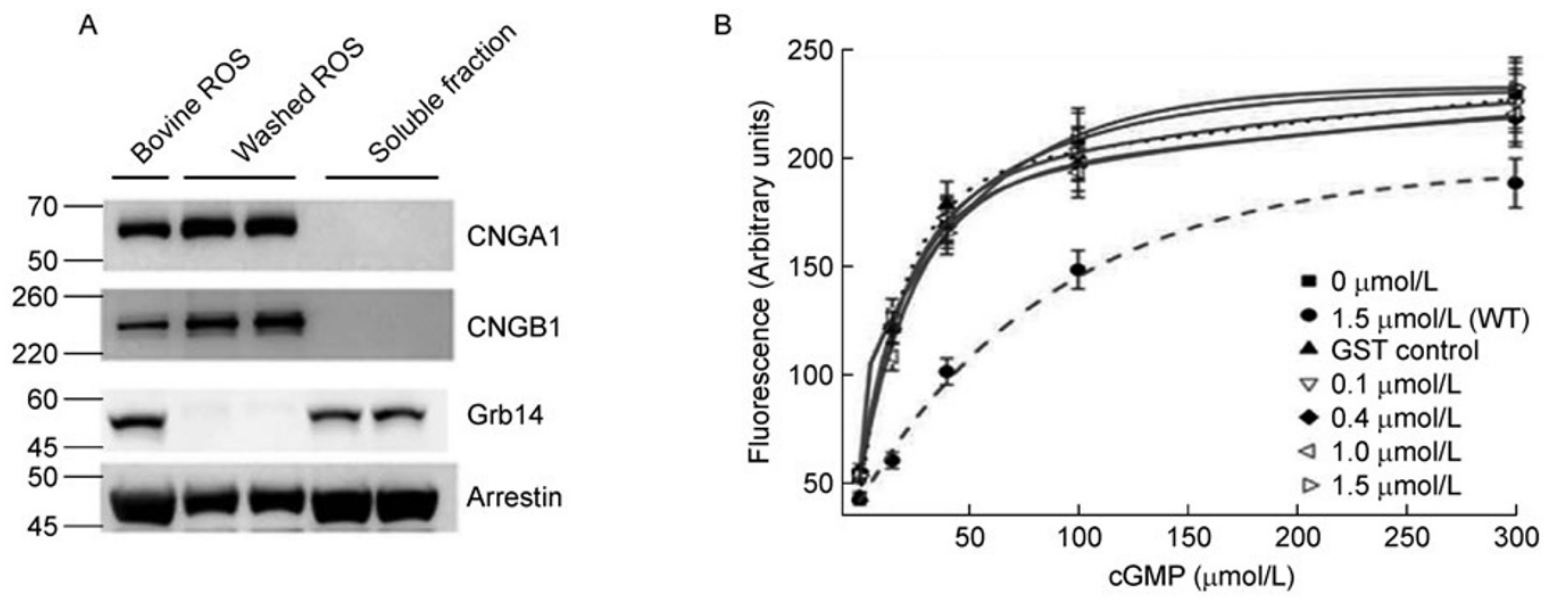

Figure 5. GST-RA-PH-Grb14 (E180-182Q) mutant domain does not modulate the $\mathrm{Ca}^{2+}$ flux across the photoreceptor membrane vesicles. (A) ROS membranes and the soluble fraction recovered after washing were subjected to immunoblot analysis with anti-CNGA1, anti-CNGB1, anti-Grb14 and anti-arrestin antibodies. (B) Vesicles were prepared from bovine ROS with trapped Fluo-3 dye. The ROS vesicles were incubated with different concentrations of mutant GST-RA-PH-Grb14 (0.1, $0.4,1.0$ and $1.5 \mu \mathrm{mol} / \mathrm{L})$ or wild type GST-RA-PH-Grb14 $(1.5 \mu \mathrm{mol} / \mathrm{L})$ as indicated and followed for the effect of cGMP-induced alteration of fluorescence at $520 \mathrm{~nm}$ as a function of $\mathrm{Ca}^{2+}$ flux across the membrane. GST is the dotted trace $(1.5 \mu \mathrm{mol} / \mathrm{L})$. Wild type control is shown as the dashed trace. The data are shown as mean $\pm \mathrm{SD}, n=3$ independent ROS vesicles.

ROS membranes (unwashed and washed) and soluble fraction recovered after washing were subjected to immunoblot analysis with anti-CNGA1, anti-CNGB1, anti-Grb14 and anti-arrestin antibodies (Fig. 5A). The washing of the ROS membranes excludes all the soluble proteins like Grb14 (Fig. 5A). It is noteworthy that washing of ROS facilitates the release of Grb14 from the ROS membranes. This makes it easier to attribute the effect to an exogenously added factor. Arrestin expression was used as a control for ROS. The channel activity experiment revealed that the mutant construct did not exhibit any dose dependent inhibition of CNG channel as shown by wild type protein (Fig. 5B). This experiment confirms that the CNG channel modulatory effect of Grb14 is mediated through binding of $(\mathrm{Glu})_{3}(180-182)$ in 
RA domain. The lack of inhibition due to lack of proper folding upon mutation of three consecutive Glu residues of the protein was ruled out by secondary structural analysis using circular dichroism studies. The far-UV CD spectrum of proteins is particularly sensitive to secondary structure and therefore useful in studying conformational changes. The farUV CD spectra of wild type and mutant proteins (E180-182Q) are presented (Fig. 6). The spectra show strong negative ellipticities at 208 and $220 \mathrm{~nm}$, suggesting that RA-PH in the native state is composed of predominantly a-helix rich regions. The spectral trace and data analysis revealed that the two proteins (wild type and mutant) did not show any alteration in their structure and their secondary structural content was not altered (Fig. 6). The overall secondary structural content was observed to be $74 \% \pm 5 \%$ for $\alpha$-helix and $11 \% \pm 5 \%$ for $\beta$-sheets respectively. Rest of the secondary structural content was attributed to the aperiodic structure constituted by random coil and $\beta$-turns. This experiment shows that Grb14-mediated inhibition of CNG channel is mediated through these three Glu residues and that lack of inhibition of CNG channel by mutant protein (E180-182Q) is not due to the unfolding of the protein.

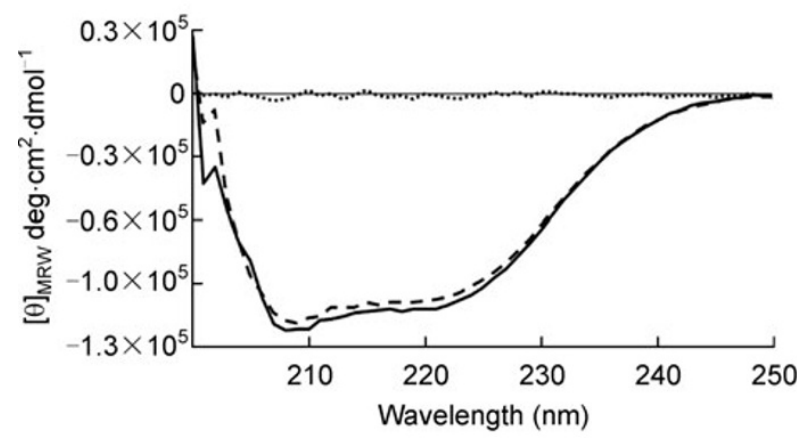

Figure 6. RA-PH-Grb14 (E180-182Q) mutation does not alter the domain secondary structure. Polyglutamate mutation does not alter the RA-PH-Grb14 secondary structure. CD spectral analysis was performed to examine the effects of polyglutamate mutation on the secondary structure of RA-PHGrb14. GST fusion proteins were expressed, purified and used in the $C D$ analysis. Representative $C D$ spectra of the wild type (solid) and mutant (E180-182Q) (dashed) after subtraction of the GST alone spectrum. Dotted line represents the buffer baseline. Quantitative analysis of the relative secondary structural content of WT and mutant proteins using CDPro showed that there is no difference between the two.

\section{Residues contributing to the binding interaction between Grb14 and CNGA1}

In order to study the residues contributing towards the strength of the physical association of the two proteins, sitedirected mutagenesis of Lys ${ }^{140}$ to Ala in Grb14 was carried out. Lys ${ }^{140}$ is a key residue which strengthens the association of Grb14 with the N-Ras (Depetris et al., 2009). Both the single mutant of Grb14 (K140A) and mutations that affect the channel modulation plus K140A (i.e. E180-182Q; K140A) resulted in the loss of association between CNGA1 and Grb 14 by $50 \% \pm 10 \%$ (Fig. 7A and 7B). These results suggest that in addition to K140, other regions in Grb14 may also be involved in the binding interaction with CNGA1.
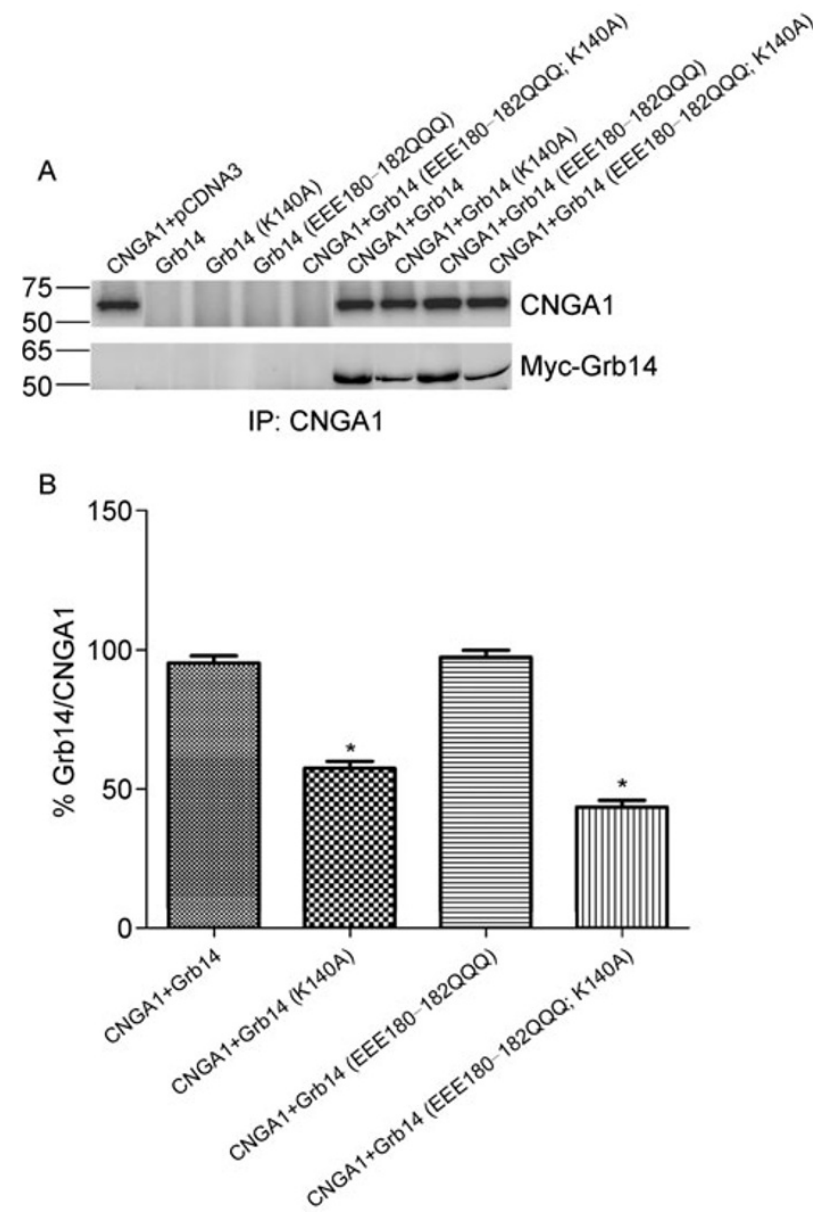

Figure 7. Molecular determinants involved in the Grb14 and CNGA1 binding. The binding between CNGA1 and wild type and mutant Grb14 proteins was investigated by subjecting the HEK293T cellular lysates expressing respective proteins to immunoprecipitation with anti-CNGA1 antibody followed by immunoblotting and probing for Grb14 with anti-myc antibody (A). The interaction was quantified by densitometric analysis of the band intensities in immunoblots, performed in the linear range of detection, normalized by the total amount of CNGA1 immunoprecipitated in each case. The relative values were expressed as percentage of the Grb14 (wild type) co-immunoprecipitated with CNGA1 (B). Significance between CNGA1+ Grb14 and CNGA1+Grb14 (K140A); and CNGA1+Grb14 (E180-182Q) and CNGA1+Grb14 (E180-182Q; K140A), $* p<0.001$. Data are mean $\pm \mathrm{SD}, n=3$. 


\section{Analysis of binding interaction and CNG channel modulation by other members of Grb7 family}

We recently reported that Grb14 modulates the CNGA1 channel activity through its RA domain in vivo (Gupta et al., 2010). Differential modulation of cardiac potassium channels by Grb7 and Grb10 has also recently been reported (Ureche et al., 2009); however the effect of Grb14 and individual domain(s) on these channels has not been studied. To determine whether Grb7 and Grb10 have any suppressive effect on CNGA1 function, HEK-293T cells were transiently co-expressed with Myc-CNGA1 and either Grb7, Grb10 or Grb14 (positive control). Protein expression was determined by immunoblotting and channel activity was determined by ratiometric measurement of $\left[\mathrm{Ca}^{2+}\right]_{i}$ in response to cGMP stimulation by means of the calcium sensitive fluorescent dye indo-1/AM (Gupta et al., 2010). No suppression of CNGA1 function was observed when channel was co-expressed with either Grb7 or Grb10 (Fig. 8A). However, a reduction in CNGA1 activity was observed when the cells were transfected with the Grb14 (Fig. 8A). The lack of suppression of intracellular $\mathrm{Ca}^{2+}$ through CNGA1 in the presence of Grb7 or Grb10 was not due to the lack of protein expression, as both of these proteins were expressed at a level comparable with Grb14 (Fig. 8B). The lack of CNGA1 suppression by either Grb7 or Grb10 could be due to their inability to interact with the CNGA1. To verify this possibility, CNGA1 immunoprecipitates were subjected to immunoblot analysis with anti-Myc antibody which detects Grb7, Grb10 and Grb14. Grb14 coimmunoprecipitated along with CNGA1 whereas Grb7 and Grb10 did not (Fig. 8C). These data suggest that even though the RA domain is highly conserved among the Grb7 family members, only Grb14 has a binding and modulatory property towards CNGA1, but not Grb7 and Grb10.

\section{DISCUSSION}

Our current study suggests that Grb14 interacts with the cGMP binding pocket of CNGA1 and suppresses the $\mathrm{Ca}^{2+}$ influx as a function of channel activity. The negatively charged cyclic phosphate ring of cGMP interacts with CNGA1-Arg ${ }^{559}$ and brings about certain conformational changes in CNGA1. The electrostatic interaction is mediated through the negatively charged E180-182Q sequence at the RA domain of the Grb14 protein. This interaction may serve as a size filter that prevents the passage of cGMP through the translocation pathway, until Grb14 protein undergoes loss of affinity for the channel following appropriate signaling events. The theoretical predictions were experimentally validated by sitedirected mutagenesis studies which further support the molecular modeling analysis. The E180-182Q mutant failed to function as wild type in modulating the CNG channel activity; however the physical interaction between the two proteins was not affected. Secondary structural investigations
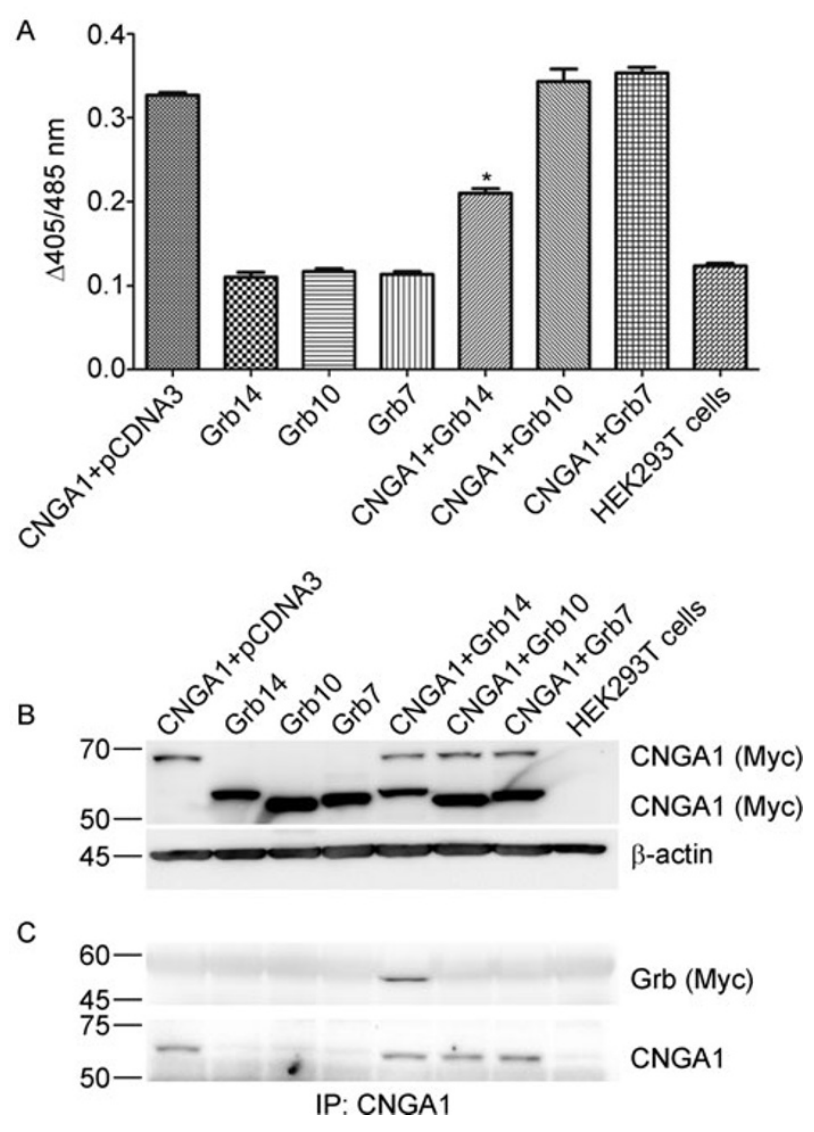

Figure 8. Analysis of binding interaction and CNG channel modulation by other members of Grb7 family. (A) CNGA1 was co-expressed along with each of the Grb14, 10 and 7 and examined for their effects on $\mathrm{Ca}^{2+}$ influx in response to cGMP. Control experiments include the expression of Grb14, 10 and 7 alone as well as CNGA1 + pcDNA3 and untransfected HEK293T cells. The data are shown as mean $\pm \mathrm{SD}, n=3$ independent transfections. $* p<0.001$ (CNGA1 vs CNGA1 plus Grb14). Protein expression in cellular lysates was determined by immunoblotting with anti-Myc (for CNGA1, Grb14) and anti- $\beta$ actin antibodies (B) or immunoprecipitated with anti-CNGA1 antibody and immunoblotted with anti-Myc antibody and reprobed with anti-CNGA1 antibody (C).

using CD spectra indicated that the lack of modulation was not due to the disruption of the secondary structure of the protein. As RA-PH constitutes a singular structural domain, therefore to rule out any experimental artifacts, a similar experiment was performed with the RA-PH domain of Grb14. The results confirmed previous observations that $\mathrm{E} 180-182 \mathrm{Q}$ was essential for CNG channel activity modulation but not for the physical binding. In addition, our study also suggests that all the three Glu residues in Grb14 are essential for the suppression of CNG channel activity. In this study we used the CNGA1 subunits alone, which have been demonstrated by us (Gupta et al., 2010) and others (Zheng et al., 2002; Zhong et al., 2003) to form functional channels in hetero- 
logous system. The data are simultaneously compared with the $\mathrm{Ca}^{2+}$ influx from native heterotetrameric CNG channels in ROS vesicles and the results are comparable. The CNGA1 and Grb14 expression levels as well as CNGA1 $\mathrm{Ca}^{2+}$ influx activity were strictly monitored to avoid faulty interpretations arising from differential protein expressions. The critical role played by photoreceptor CNG channels necessitates their efficient regulation. The native rod CNG channels are heterotetramers of 3 CNGA1 and a CNGB1 subunit. It is possible that the Grb14 interacts with one or more of the subunits and competes out the cGMP in vivo. Consistent with this hypothesis that co-immunoprecipitation of Grb14 always brings down both the subunits of (CNGA1 and CNGB1) the channel (Gupta et al., 2010). This process may further alter the allosteric co-operativity between the various subunits thereby promoting the channel to be in an off state.

In this study, cGMP-stimulated $\mathrm{Ca}^{2+}$ transport across the vesicles or cell membranes was used instead of individual channel recordings to study the global impact of this proteinprotein interaction on a model close to physiological system and to minimize the errors arising from individual electrophysiological recordings. This also helped us avoid effects arising out of any altered trafficking or expression of CNG channels or Grb14. This highly sensitive method helped us quantify the channel inhibition levels.

The co-expression data along with the results presented on the inhibition of native channel by recombinant RA domain show that Grb14 did not affect the level of CNGA1 expression in HEK-293T cells, indicating that Grb14 modulates the CNG channel function rather than affecting its expression. The inhibition of native channel by recombinant RA domain also argues against that co-expressing wild-type CNGA1 with Grb14 leads to a change in trafficking in HEK-293T cells that results in fewer channels in the plasma membrane. The $\mathrm{Ca}^{2+}$ release from ROS vesicles and HEK-293T cells in response to cGMP stimulation is comparable, further suggesting that Grb14 modulates CNG channel function.

It has been recently shown that Lys ${ }^{140}$ is a critical residue for the interaction of Grb14 with N-Ras (Depetris et al., 2009). Our studies suggest that substitution of Lys 140 for Ala in the RA domain of Grb14 resulted in 50\% less binding to Cterminal domain of CNGA1. This signifies a similar interaction pattern between Grb14 and N-Ras on the one hand and Grb14 and C-terminal tail of CNGA1 on the other, strengthening the fact that the folding pattern of cytoplasmic C-terminal domain of CNGA1 exhibits similarity towards the Ras proteins (data not shown). This also highlights the diversity of the amino acid constraints contributing towards CNGA1 activity modulation and the potency of physical binding. Our results also suggest that in addition to Lys 140, other regions in Grb14 may also be involved in the physical association with CNGA1.

The binding interaction between Grb14 and CNG channel suggests that Grb14 might also play a role in regulating other ion channels perhaps through nucleotide-binding sites pre- sent in their cytosolic domains. It is interesting to note that E180-182 region is only present in Grb14 and is absent in Grb7 and Grb10 (Daly et al., 1996). Grb7 and Grb10 have previously been shown to differently modulate the cardiac potassium channels (Ureche et al., 2009). It is tempting to speculate that Grb7 and Grb10 may regulate other ion channels (Ureche et al., 2009), whereas Grb14 could be more specific towards cyclic nucleotide-gated channels. Interestingly, in retinal rod photoreceptors only Grb14 is expressed but not Grb7 and Grb10 (authors unpublished data). Our studies further suggest the existence of divergence in RA domains among the members of the Grb7 family, and that Grb14 may have been evolved later in the evolution that binds to both Ras and nucleotide binding proteins. Further studies are required to establish these findings.

Moreover, our studies indicate that Grb14 may be a molecular switch that regulates CNGA1 activity with respect to cGMP availability and accessibility to the active site. Thus the Glu residues on the $\beta$-turn may comprise the signature sequence of RA-Grb14 that regulates the CNGA1 channel. The role of this signature sequence in other RA domains needs to be investigated. A limitation of this model is that the relative structure and position of one domain with respect to others in a multidomain protein cannot be easily predicted in the absence of other information. However, the structure of functionally conserved binding sites may be predicted most accurately. The models presented here for the Grb14 RA domain interaction with CNGA1 may not be correct in all details; however the specific electrostatic interactions between the two proteins has been tested by site-directed mutagenesis. Consistent with our hypothesis, elimination of these interactions terminates the Grb14-induced modulation of CNGA1, thereby increasing CGMP accessibility and sensitivity of CNGA1.

In summary, our results demonstrate that Grb14 is a physiological modulator of channel activity in light and binds to CTR of CNGA1 through its RA domain and modulates the channel activity. These studies provide evidence that Grb14 is a normal physiological modulator of the CNG channel. Understanding the precise molecular mechanism of Grb14mediated inhibition of the rod CNG channel will provide the foundation to design novel therapeutic agents to modulate the channel in retinal degenerative diseases. These findings may have significance in other ions channels and should have a broad applicability to other neuronal cells. Further studies are required to understand the functional interaction between Grb14 and CNG channel in rod cell physiology and these studies are currently underway in our laboratory.

\section{MATERIALS AND METHODS}

\section{Cell lines and culture condition}

HEK 293T cells were maintained in DMEM medium at $37^{\circ} \mathrm{C}$. 
Approximately $2.5 \times 10^{5}$ cells were seeded in each culture dish $12-18 \mathrm{~h}$ before transfection. Calcium phosphate-mediated DNA transfection was performed using each of the plasmids containing the cDNA of interest (Wigler et al., 1978) and cells were harvested for experiments about $48 \mathrm{~h}$ posttransfection.

\section{Plasmids and vectors}

Myc-tagged bovine Grb14 (wild type) construct was cloned as described previously (Rajala et al., 2009). E180Q; E182Q; E180-181Q; and E180-182Q mutants of the full length as well as RA and RA-PH Grb14 were generated by site-directed mutagenesis (Rajala et al., 2004). The RA (residues 105-195) and RA-PH (residues 105-355) domains of Grb14 were cloned into the Myctagged pCDNA3 vector as described previously (Gupta et al., 2010). E180-182Q mutants of the full length as well as RA and RA-PH Grb14 were generated by site-directed mutagenesis. Myc-tagged CNGA1 (wild type) construct was generated from the amplification of pCDNA3-CNGA1 as described previously (Gupta et al., 2010). Sitedirected mutagenesis of the wild type CNGA1 was carried out to generate R559Q mutant. The primers used for SDM are CNGA1R559Q (sense: GCTGGCAATCGACAAACAGCCAATATTAAA; antisense: TTTAATATTGGCTGTTTGTCGATTGCCAGC), and Grb14E180-182Q (sense: AACTGGGGAATG CAACAACAAAATAAGCTATACTTT; antisense: AAAGTATAGCTTATTTTGTTGTTGCATTCCCCAGTT). After sequencing, the mutant CDNAs were excised from the sequencing vector and cloned into pCDNA3 mammalian expression vector.

\section{Assessment of the channel activity by ratiometric measurement of $\left[\mathrm{Ca}^{2+}\right]_{i}$}

The fluorescent indicator Indo-1/AM was used to monitor $\mathrm{Ca}^{2+}$ influx through the CNGA1 channels in cell suspensions. The assays were performed as described (Grynkiewicz et al., 1985) using a spectrofluorometer (Fluostar Omega, BMG lab tech $\mathrm{GmBH}$, Offenburg, Germany). This assay was designed to determine CNG channel activity in cell populations $\left(2 \times 10^{6}\right)$ in response to 8 -pCPT-cGMP stimulation. Briefly, cells (36-48 h posttransfection) were harvested with cell dissociation medium (Invitrogen, Carlsbad, CA), washed with the extracellular solution (ECS; $140 \mathrm{mmol} / \mathrm{L} \mathrm{NaCl}, 5 \mathrm{mmol} / \mathrm{L} \mathrm{KCl}$, $1 \mathrm{mmol} / \mathrm{L} \mathrm{MgCl} 2,1.8 \mathrm{mmol} / \mathrm{L} \mathrm{CaCl} 2,10 \mathrm{mmol} / \mathrm{L}$ glucose, $15 \mathrm{mmol} / \mathrm{L}$ HEPES, $\mathrm{pH} 7.4$ ), and incubated with $2 \mu \mathrm{mol} / \mathrm{L}$ Indo-1/AM (SigmaAldrich) in ECS in the presence of $0.05 \%$ Pluronic F-127 (Invitrogen, Carlsbad, CA) for $40 \mathrm{~min}$ at room temperature. Then, the cells were washed three times with ECS and resuspended in ECS $\left(1 \times 10^{6} / \mathrm{mL}\right)$. $\mathrm{Ca}^{2+}$ influx in response to 8-pCPT-cGMP was determined by ratiometric measurement, which represents the free intracellular $\mathrm{Ca}^{2+}$ concentration. Changes of intracellular $\mathrm{Ca}^{2+}$ concentration were expressed as a $\Delta 405 / 485$ ratio. Data were analyzed and graphed using GraphPad Prism software (GraphPad Software, San Diego, CA).

\section{Expression of GST-fusion proteins}

An overnight culture of $E$. coli BL21 (DE3) (pGEX-RA-PH and pGEXRA-PH (E180-182Q) was diluted 1:10 with LB containing $100 \mu \mathrm{g} / \mathrm{mL}$ ampicillin grown for $1 \mathrm{~h}$ at $37^{\circ} \mathrm{C}$, and induced for another hour by addition of IPTG to $1 \mathrm{mmol} / \mathrm{L}$. Bacteria were sonicated three times for
$20 \mathrm{~s}$ each time in lysis buffer containing $50 \mathrm{mmol} / \mathrm{L}$ HEPES $(\mathrm{pH} 7.5)$, $50 \mathrm{mmol} / \mathrm{L} \mathrm{NaCl}, 10 \%$ glycerol, $1 \%$ Triton X-100, $1 \mathrm{mmol} / \mathrm{L}$ EDTA, $1 \mathrm{mmol} / \mathrm{L}$ EGTA, $10 \mathrm{mg} / \mathrm{mL}$ aprotinin, $10 \mathrm{mg} / \mathrm{mL}$ leupeptin, and $1 \mathrm{mmol} / \mathrm{L}$ phenylmethylsulfonyl fluoride (PMSF). Lysates were clarified by centrifugation, and the supernatants were incubated with $500 \mu \mathrm{L}$ of $50 \%$ gluthathione-coupled beads (Amersham Pharmacia) for $30 \mathrm{~min}$ at $4^{\circ} \mathrm{C}$. The GST fusion proteins were washed in PBS $\left(137 \mathrm{mmol} / \mathrm{L} \mathrm{NaCl}, 2.7 \mathrm{mmol} / \mathrm{L} \mathrm{KCl}, 4.3 \mathrm{mmol} / \mathrm{L} \mathrm{Na}{ }_{2} \mathrm{H}-\right.$ $\mathrm{PO}_{4} \cdot 7 \mathrm{H}_{2} \mathrm{O}$, and $\left.1.4 \mathrm{mmol} / \mathrm{L} \mathrm{KH}_{2} \mathrm{PO}_{4}\right)$ and eluted twice with $1 \mathrm{~mL}$ of $5 \mathrm{mmol} / \mathrm{L}$ reduced glutathione (Sigma) in $10 \mathrm{mmol} / \mathrm{L}$ Tris- $\mathrm{HCl}(\mathrm{pH} 7.4)$ and aliquots of fusion proteins were stored at $-20^{\circ} \mathrm{C}$.

\section{Assessment of the channel activity in ROS vesicles}

The effect of Grb14 on CNG channel activity was investigated using Fluo-3 a fluorometric calcium ion assay (Gupta et al., 2010). Bovine ROS was prepared on a continuous sucrose gradient and washed twice in buffer (Hsu and Molday, 1993) that removes Grb14, which is a soluble protein from the ROS. The washed membranes $(19 \mathrm{mg} / \mathrm{mL})$ were suspended in buffer containing $10 \mu \mathrm{mol} / \mathrm{L}$ Fluo-3, a fluorescent $\mathrm{Ca}^{2+}$ indicator, and sonicated (Gupta et al., 2010). The sonicated ROS membranes were then extruded three times through a lipid extruder containing two layers of nucleopore polycarbonate membranes with pore sizes of 400 and $200 \mathrm{~nm}$. The vesicles were dialyzed extensively at $4^{\circ} \mathrm{C}$ for $6 \mathrm{~h}$ to exclude untrapped dye. The whole experiment was carried out in the dark to minimize the light bleaching effect on Fluo-3. The sample was diluted and incubated with various concentrations of exogenously added wild type or mutant GST-RA$\mathrm{PH}-\mathrm{Grb} 14$, or GST $(5 \mu \mathrm{mol} / \mathrm{L})$ as indicated and the $\mathrm{Ca}^{2+}$ influx assay performed as described previously (Gupta et al., 2010).

\section{Secondary structural analysis}

CD spectra were collected with a JASCO J-500A (Japan Spectroscopic Co., Tokyo, Japan) automatic recording spectropolarimeter equipped with a $500 \mathrm{~N}$ data processor, xenon lamp and a PTC-348WI Peltier uniform temperature cell holder. Dry nitrogen was purged continuously before and during the experiment. Conformational changes in the secondary structure of the protein $(0.3 \mu \mathrm{g} / \mu \mathrm{L})$ in $50 \mathrm{mmol} / \mathrm{L}$ Tris- $\mathrm{HCl}$ buffer, $\mathrm{pH} 7.4$, at $27^{\circ} \mathrm{C}$ were monitored in the farUV region between 190 and $250 \mathrm{~nm}$ in the cuvette with a path length of $1 \mathrm{~mm}$. After appropriate buffer and GST blanks were run and subtracted, mean residue ellipticities were calculated using the equation $[\theta]=\theta_{\mathrm{obs}} \mathrm{MRW} / c \mathrm{l}$, where $\theta_{\mathrm{obs}}$ is the observed ellipticity in degrees, MRW is the mean residue weight, $c$ is the concentration of protein $(\mathrm{mg} / \mathrm{mL})$ and $/$ is the path length in centimeters. A mean residue molecular weight of 110 and sensitivity of $1 \mathrm{~m} / \mathrm{cm}$ (millidegree per centimeter) was used with a scan speed of $10 \mathrm{~nm} / \mathrm{min}$ using a bandwidth of $1 \mathrm{~nm}$. An average of three runs was recorded for each sample. The secondary structural changes were analyzed by the method as described (Yang et al., 1986). Protein secondary structural content was estimated using CDPro (Sreerama and Woody, 2000) and the resulting calculations were averaged to yield the percent of relative secondary structural content.

\section{Molecular modeling studies}

The protein tertiary structural modeling was done using program MODELLER (Eswar et al., 2008). RA domain of Grb14 [UniProtKB 
Accession No. Q5ICW4] (106-192) was constructed using the RA domain of Grb10 as the template (PDB ID: 3HK0). Ribbon model of CNGA1 (residues 400-600) was constructed based on template [PDB ID. 1Q3E] and its topological analysis performed using the public domain topology prediction program (http://expasy.org/tools/ \#topology). Structural superimpositions and graphical manipulations were performed using Arguslab (Thompson, Planaria software), VMD (Humphrey et al., 1996) and DaliLite v3 (Holm et al., 2008). The extensive energy minimization was done using Deep View (Guex and Peitsch, 1997). The energy calculations were performed using the universal force field method (UFF) of molecular mechanics. All the energy calculations were performed at a net molecular charge of zero and a medium dielectric constant of 15 . The geometrical optimization of the modeled protein was performed by initial 150 steps of Simplex minimization. This was further followed by a steepest descent minimization to achieve a root-mean-square (RMS) force of 1.5 $\mathrm{kcal} / \mathrm{mol} \cdot \AA^{-1}$, and subsequently a conjugate gradient minimization (CGM) to achieve an RMS force of $0.5 \mathrm{kcal} / \mathrm{mol} \cdot \AA^{-1}$. A few knotty side-chain conformations were acknowledged and rectified. Structural parameters and prediction quality of the modeled structure were evaluated based on bond angle stereo-chemistry using WHATIF (Vriend, 1990) and PROCHECK (Morris et al., 1992).

\section{Statistical methods}

Data were analyzed and graphed using Graphpad Prism software (GraphPad Software, San Diego, CA). The data were expressed as the mean \pm SD and compared by Student's $t$ test for unpaired data. The significance was set at $p<0.05$.

\section{ACKNOWLEDGEMENTS}

This work was supported by grants from the NIH (EY016507; EY00871; EY12190). CNGA1 and CNGB1 subunit channel antibodies were a kind gift from Dr. Robert Molday, University of British Columbia (Canada).

\section{ABBREVIATIONS}

CNG, cyclic nucleotide gated channel; CTR, C-terminal region; Grb14, growth factor receptor-bound protein 14; RA, Ras-associating domain; ROS, rod outer segments

\section{REFERENCES}

Biel, M., Zong, X., Ludwig, A., Sautter, A., and Hofmann, F. (1999). Structure and function of cyclic nucleotide-gated channels. Rev Physiol Biochem Pharmacol 135, 151-171.

Daly, R.J. (1998). The Grb7 family of signalling proteins. Cell Signal 10, 613-618.

Daly, R.J., Sanderson, G.M., Janes, P.W., and Sutherland, R.L. (1996). Cloning and characterization of GRB14, a novel member of the GRB7 gene family. J Biol Chem 271, 12502-12510.

Depetris, R.S., Wu, J., and Hubbard, S.R. (2009). Structural and functional studies of the Ras-associating and pleckstrin-homology domains of Grb10 and Grb14. Nat Struct Mol Biol 16, 833-839.

Eswar, N., Eramian, D., Webb, B., Shen, M.Y., and Sali, A. (2008).
Protein structure modeling with MODELLER. Methods Mol Biol 426, 145-159.

Finn, J.T., Grunwald, M.E., and Yau, K.W. (1996). Cyclic nucleotidegated ion channels: an extended family with diverse functions. Annu Rev Physiol 58, 395-426.

Grynkiewicz, G., Poenie, M., and Tsien, R.Y. (1985). A new generation of $\mathrm{Ca} 2+$ indicators with greatly improved fluorescence properties. J Biol Chem 260, 3440-3450.

Guex, N., and Peitsch, M.C. (1997). SWISS-MODEL and the SwissPdbViewer: an environment for comparative protein modeling. Electrophoresis 18, 2714-2723.

Gupta, V.K., Rajala, A., Daly, R.J., and Rajala, R.V. (2010). Growth factor receptor-bound protein 14: a new modulator of photoreceptor-specific cyclic-nucleotide-gated channel. EMBO Rep 11, 861-867.

Holm, L., Kääriäinen, S., Rosenström, P., and Schenkel, A. (2008). Searching protein structure databases with DaliLite v.3. Bioinformatics 24, 2780-2781.

Holt, L.J., and Siddle, K. (2005). Grb10 and Grb14: enigmatic regulators of insulin action-and more? Biochem J 388, 393-406.

Hsu, Y.T., and Molday, R.S. (1993). Modulation of the cGMP-gated channel of rod photoreceptor cells by calmodulin. Nature 361 , 76-79.

Humphrey, W., Dalke, A., and Schulten, K. (1996). VMD: visual molecular dynamics. J Mol Graph 14, 33-38, 27-28.

Kaupp, U.B., and Seifert, R. (2002). Cyclic nucleotide-gated ion channels. Physiol Rev 82, 769-824.

Kumar, V.D., and Weber, I.T. (1992). Molecular model of the cyclic GMP-binding domain of the cyclic GMP-gated ion channel. Biochemistry 31, 4643-4649.

Liu, D.T., Tibbs, G.R., Paoletti, P., and Siegelbaum, S.A. (1998). Constraining ligand-binding site stoichiometry suggests that a cyclic nucleotide-gated channel is composed of two functional dimers. Neuron 21, 235-248.

Morris, A.L., MacArthur, M.W., Hutchinson, E.G., and Thornton, J.M. (1992). Stereochemical quality of protein structure coordinates. Proteins 12, 345-364.

Rajala, A., Daly, R.J., Tanito, M., Allen, D.T., Holt, L.J., Lobanova, E. S., Arshavsky, V.Y., and Rajala, R.V. (2009). Growth factor receptor-bound protein 14 undergoes light-dependent intracellular translocation in rod photoreceptors: functional role in retinal insulin receptor activation. Biochemistry 48, 5563-5572.

Rajala, R.V., McClellan, M.E., Chan, M.D., Tsiokas, L., and Anderson, R.E. (2004). Interaction of the retinal insulin receptor beta-subunit with the p85 subunit of phosphoinositide 3-kinase. Biochemistry $43,5637-5650$.

Sreerama, N., and Woody, R.W. (2000). Estimation of protein secondary structure from circular dichroism spectra: comparison of CONTIN, SELCON, and CDSSTR methods with an expanded reference set. Anal Biochem 287, 252-260.

Ureche, O.N., Ureche, L., Henrion, U., Strutz-Seebohm, N., Bundis, F., Steinmeyer, K., Lang, F., and Seebohm, G. (2009). Differential modulation of cardiac potassium channels by Grb adaptor proteins. Biochem Biophys Res Commun 384, 28-31.

Vriend, G. (1990). WHAT IF: a molecular modeling and drug design program. J Mol Graph 8, 52-56, 29.

Wigler, M., Pellicer, A., Silverstein, S., and Axel, R. (1978). Biochemical transfer of single-copy eucaryotic genes using total 
cellular DNA as donor. Cell 14, 725-731.

Yang, J.T., Wu, C.S., and Martinez, H.M. (1986). Calculation of protein conformation from circular dichroism. Methods Enzymol 130, 208-269.

Yau, K.W., and Baylor, D.A. (1989). Cyclic GMP-activated conductance of retinal photoreceptor cells. Annu Rev Neurosci 12, 289-327.

Yau, K.W., and Hardie, R.C. (2009). Phototransduction motifs and variations. Cell 139, 246-264.

Zhang, Y., Molday, L.L., Molday, R.S., Sarfare, S.S., Woodruff, M.L.,
Fain, G.L., Kraft, T.W., and Pittler, S.J. (2009). Knockout of GARPs and the beta-subunit of the rod cGMP-gated channel disrupts disk morphogenesis and rod outer segment structural integrity. J Cell Sci 122, 1192-1200.

Zheng, J., Trudeau, M.C., and Zagotta, W.N. (2002). Rod cyclic nucleotide-gated channels have a stoichiometry of three CNGA1 subunits and one CNGB1 subunit. Neuron 36, 891-896.

Zhong, H., Lai, J., and Yau, K.W. (2003). Selective heteromeric assembly of cyclic nucleotide-gated channels. Proc Natl Acad Sci U S A 100, 5509-5513. 